\title{
An engraved surface induces weak adherence and high proliferation of nonadherent cells and microorganisms during culture
}

\section{Sunil Thomas*,1 (iD)}

${ }^{1}$ Lankenau Institute for Medical Research, Wynnewood, PA 19096, USA; *Author for correspondence: suntom2@gmail.com

BioTechniques 69: 113-125 (August 2020) 10.2144/btn-2020-0022

First draft submitted: 4 March 2020; Accepted for publication: 21 May 2020; Published online: 12 June 2020

\section{ABSTRACT}

When cells are cultured in a Petri dish, the adherent cells attach to the bottom of the dish; whereas, the nonadherent cells float in the culture medium. It was observed that nonadherent cells could be induced to adherent-like cells when cultured in an engraved plastic dish (biosimulator). The adherence of these cells to the engraved surface could be prevented with inhibitors specific for adhesion. It was also observed that culturing microorganisms of the environment in a biosimulator induced weak adhesion and high proliferation. Analysis of the microbiome using $16 \mathrm{~S}$ rRNA profiling demonstrated that the biosimulator was more efficient in inducing proliferation of several phyla of microorganisms compared with culture by conventional techniques.

\section{METHOD SUMMARY}

Nonadherent cells could be induced to adherent-like cells when cultured in an engraved plastic dish (biosimulator). The adhesion induced proliferation of eukaryotic and prokaryotic cells.

\section{KEYWORDS:}

adherence $\bullet$ biosimulator $\bullet$ cell culture $\bullet$ diagnostics $\bullet$ engraving $\bullet$ environment $\bullet$ microbiome $\bullet$ microorganisms $\bullet$ nonadherent cells - Petri dish

Cell culture is routinely employed in laboratories for biomedical research and has multiple applications, including diagnosis, drug development, therapy and production of biological resources. The traditional process of culturing adherent cells in a Petri dish still serves as an important tool for cell culture applications [1]. During cell culture, the adherent cells attach to the bottom of a plastic Petri dish, whereas nonadherent cells float in the culture medium. There are no specialized dishes for transforming nonadherent cells to adherent cells; this is an impediment in several drug development studies, especially in those involving autoreactive T cells or cells involved in atherosclerosis.

The majority of microorganisms of the environment are nonculturable [2]. In addition, some microorganisms exist in a viable but nonculturable state and are difficult to culture in laboratory conditions. Such cells are characterized by a lack of culturability using conventional culture techniques that impairs their detection by routine analytical techniques. This leads to an underestimation of total viable cells in environmental or clinical samples, and thereby poses a risk to public health [3]. Hence, there is a need to develop a strategy to increase the proliferation of such microorganisms.

In this paper, it is demonstrated that nonadherent cells exhibit adherence when cultured in an engraved polystyrene Petri dish. Though the cells cultured were from the same clone, when cultured on an engraved dish, they exhibited polarity. It was also demonstrated that specific inhibitors could be used to prevent adhesion of nonadherent cells on an engraved surface. Further, we demonstrate that the biosimulator enhanced the proliferation of microorganisms. The phenomenon of cell adhesion on an engraved dish has wide applications in cell biology and microbiology.

\section{Materials \& methods}

Development of a biosimulator to induce adhesion \& proliferation of cells

For culturing cells, nonpyrogenic, noncytotoxic and polystyrene BioLite cell culture dishes (Thermo Scientific, NJ, USA) were used. To induce adhesion of cells, the plastic surface was engraved with parallel lines using a sterile sharp blade under aseptic conditions. The engravings had a width of 30-50 $\mu \mathrm{m}$ and depth of $5 \mu \mathrm{m}$. 


\section{Cell culture in the biosimulator}

The nonadherent hybridoma cell lines 4B7, 10D9, 1A10, 99D, Sp2/0, B56T $\left(1 \times 10^{6}\right.$ cells/dish) were cultured in $10 \mathrm{ml}$ Dulbecco's modified Eagle medium, and incubated at $37^{\circ} \mathrm{C}, 5 \% \mathrm{CO}_{2}$. The cells were monitored daily. All the experiments were repeated at least three times.

For cell proliferation assays, hybridoma cells were grown in a conventional dish or a biosimulator. Cells were collected every other day, depending on the proliferation. The absorption of cells was measured at $600 \mathrm{~nm}$ using a spectrophotometer; a hemocytometer was used for cell count.

For inhibition of cell adhesion, $0.1 \%$ salicylic acid (Fisher Scientific, NJ, USA) and $0.01 \%$ pectasol (gift of ecoNugenics, CA, USA) were added to the culture medium during cell culture.

\section{Proliferation \& analyses of microorganisms in a biosimulator}

Samples from soil, spring water, air (office) and host oral microbiome were cultured in a biosimulator in Luria-Bertani medium for $48 \mathrm{~h}$. After $48 \mathrm{~h}$, the media were pelleted in a centrifuge at $5000 \times \mathrm{g}$ and the samples subjected to $16 \mathrm{~S}$ rRNA sequencing (Arizona State University Microbiome Core) for taxonomic identification. Microbial DNA was extracted from samples using the DNeasy PowerSoil Kit (Qiagen, Germany) following the manufacturer's directions.

\section{Microbiome library preparation methodology}

Bacterial community analysis was performed via next generation sequencing on the Illumina MiSeq platform. Amplicon sequencing of the V4 region of the 16S rRNA gene was performed with the barcoded primer set 515f/806r designed by Caporaso et al. [4] and following the protocol by the Earth Microbiome Project (http://www.earthmicrobiome.org/emp-standard-protocols/) for library preparation. PCR amplifications for each sample were done in triplicate, then pooled and quantified using the Quant-i $T^{T M}$ PicoGreen ${ }^{\circledR}$ dsDNA Assay Kit (Invitrogen, CA, USA). A no-template control sample was included during library preparation as a control for extraneous nucleic acid contamination. About $240 \mathrm{ng}$ of DNA per sample were pooled and then cleaned using the QIAquick PCR purification kit (Qiagen). The pool was quantified using the Illumina library Quantification Kit ABI Prism ${ }^{\circledR}$ (Kapa Biosystems, MA, USA), then diluted to a final concentration of $4 \mathrm{nM}$, denatured, and diluted to a final concentration of $4 \mathrm{pM}$ with $15 \%$ of PhiX control library (Illumina, CA, USA). Finally, the DNA library was loaded in the MiSeq and run using the version 2 module, $2 \times 250$ paired end, following the directions of the manufacturer.

Analysis of sequencing results was performed by the University of North Carolina Microbiome Core. The sequencing output from the MiSeq platform was converted to FASTQ format and demultiplexed using Bcl2Fastq 2.18.0.12 (Illumina). The resulting paired-end reads were processed using QIIME 2 2018.11. Index and linker primer sequences were trimmed using the QIIME 2 invocation of cutadapt. The resulting paired-end reads were processed with DADA2 through QIIME 2, including merging paired ends, quality filtering, error correction and chimera detection.

Amplicon sequencing units from DADA2 were assigned taxonomic identifiers according to Green Genes release 13_08, the Human Oral Microbiome Database release 15.1 and Silva database release 132.

Alpha diversity with respect to Faith PD whole tree, Evenness (Shannon) index and observed species number metrics was estimated using QIIME 2 at a rarefaction depth of 5000 sequences per subsample. Beta diversity estimates were calculated within QIIME 2 using weighted and unweighted Unifrac distances as well as Bray-Curtis dissimilarity between samples at a subsampling depth of 5000 . Results were summarized, visualized through principal coordinate analysis and significance was estimated as implemented in QIIME 2. Significance of differential abundance was estimated using AnCom as implemented in QIIME 2.

\section{Statistics}

Unpaired two-tailed Student $t$-tests were used to compare sets of data obtained from independent groups. Statistical significance was considered at the $\mathrm{p}<0.05$ level.

\section{Results \& discussion}

\section{An engraved surface could induce adhesion of nonadherent cells}

Based on the observation that a cell culture plate with a compromised surface has high affinity for cells, parallel lines were engraved on a tissue culture Petri dish and used the dish for cell culture. The cells were nonadherent and were found floating on the first and second day of culture. After 3 days of culture, all the cell lines tested (4B7, 10D9, 1A10, 99D, Sp2/0,99D) formed distinct patterns on the engraved plastic surface; pattern formation corresponded to the engraving. When the Petri dish was engraved with parallel lines, the nonadherent cells were seen on top of the engraved line, whereas on the lower half of the dish the nonadherent cells were below the engraved line (Figure 1). The orientation of the cells is shown in Figure 1C \& D. The cells were closely packed on the engraved line. The alignment of nonadherent cells was found to be stable on the engraved line; minor disturbances to the plastic dish did not destroy the patterns exhibited. This failure to destroy the patterns demonstrated that the cells were adhered near the engraved surface. The experiment demonstrated that an engraved surface could induce nonadherent cells to become adherent cells.

In conventional cell culture, adherent cells grow to confluence, and must be dissociated from the dish using trypsin. In our experiment, the nonadherent cells (unlike the adherent cells) were not strongly adhered to the biosimulator. Liquid media were pipetted to disrupt 


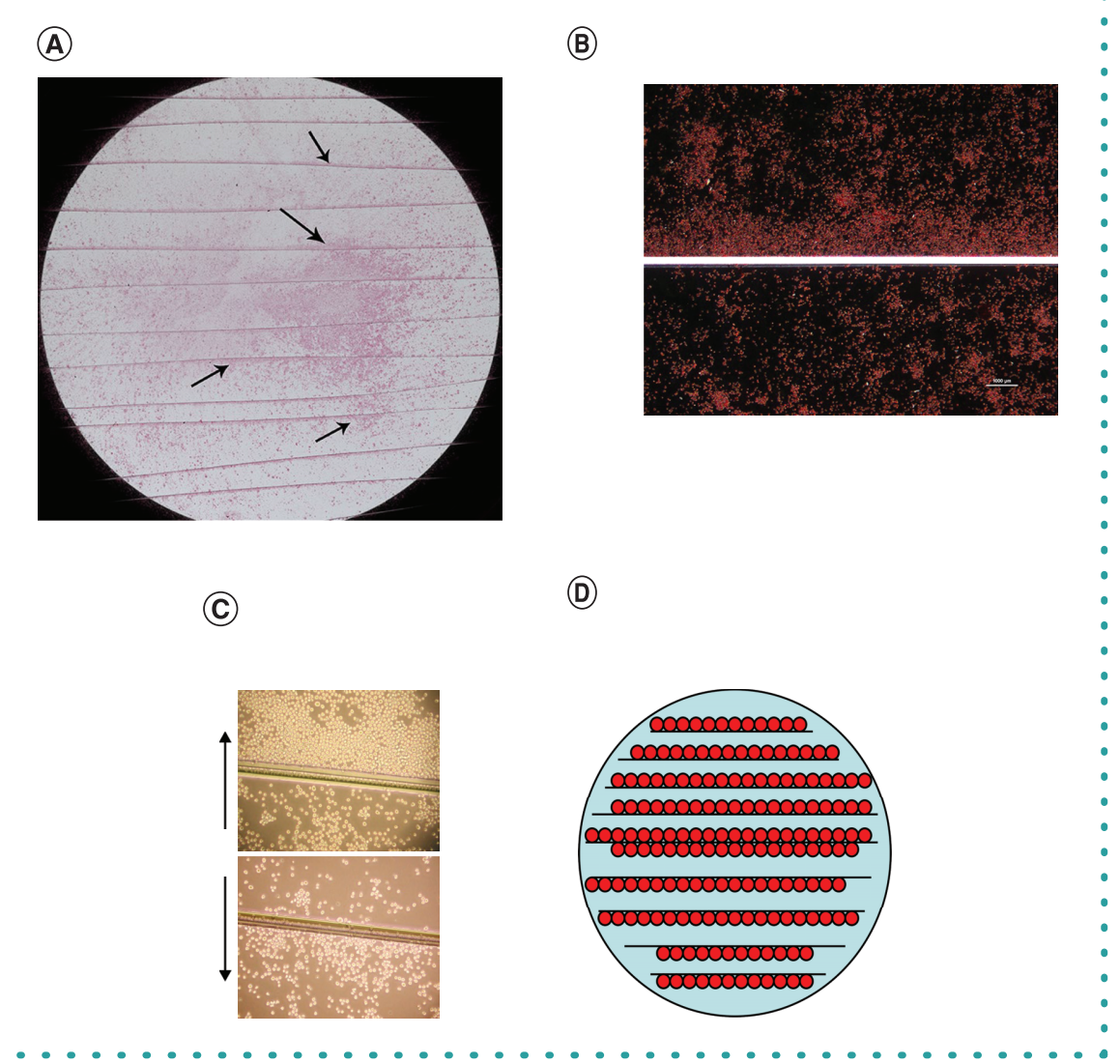

Figure 1. Engraved surface-induced adhesion of nonadherent cells. (A) Photomicrograph of nonadherent cells (4B7) exhibiting polarity on an engraved dish (biosimulator). (B) Ponceau S staining of cells cultured in a biosimulator. (C) The orientation of cells in a biosimulator. Cells are aligned on top of the engraving in the upper half of the biosimulator, whereas they are aligned below the engraving in the bottom half of the biosimulator. (D) Drawing of an engraved Petri dish showing the orientation of nonadherent cells. Experiments were repeated five times.

the cells from a distance of $1 \mathrm{~cm}$. Seventy percent of the cells were removed when the media was pipetted at a speed of $5 \mathrm{ml}$ per $10 \mathrm{~s}$ (repeated five times). The experiment demonstrated that the nonadherent cells are weakly adhered to the engraving of the biosimulator.

\section{An engraved surface induced proliferation of nonadherent cells}

We determined whether the engraved surface induced proliferation of nonadherent cells. B cell hybridoma cell lines 99D (Figure 2) and 4B7 (Figure 3) were cultured in an engraved biosimulator, using conventional Petri dishes as a control. We counted the cells and also determined the absorbance (optical density at $600 \mathrm{~nm}$ ) at regular intervals. The biosimulator induced proliferation of the nonadherent cells, as determined by cell counting and absorbance measurements (Figures $2 \& 3$ ). Culture of nonadherent cells in a biosimulator led to a statistical increase in the number of cells compared with culturing in conventional Petri dishes. The study demonstrated that engraving patterns in a biosimulator could induce adhesion and proliferation of nonadherent cells.

\section{Adhesion of nonadherent cells in a biosimulator could be inhibited by specific inhibitors}

Salicylic acid is known to prevent cell-cell interaction and is used in animal models of diabetes [5], but its mechanism of action is not clearly known. When nonadherent cells (4B7) were treated with $0.1 \%$ salicylic acid, cell adhesion was inhibited (Figure 4). We also used the adhesion inhibitor pectasol (which prevents cancer metastasis) in our studies [6]. Treatment of nonadherent cells with $0.01 \%$ pectasol did not prevent cell proliferation; however, it prevented cells adhering to the plastic surface (Figure 4C). The nonadherent cells lost the orientation property; the cells were found floating in the medium and did not have any affinity for the engraved surface. These in vitro experiments demonstrate that the phenomenon of pattern formation could be used in drug discovery studies.

\section{The engraved surface-induced proliferation of microorganisms}

As the engraved surface in the biosimulator could induce cell proliferation, we were interested to determine whether it could also induce proliferation of microorganisms. One of the impediments in microbial studies or diagnosis is the nonculturable property of most of the microorganisms. Only a very limited number of microorganisms are culturable by conventional culture techniques; there is therefore a 
(A)

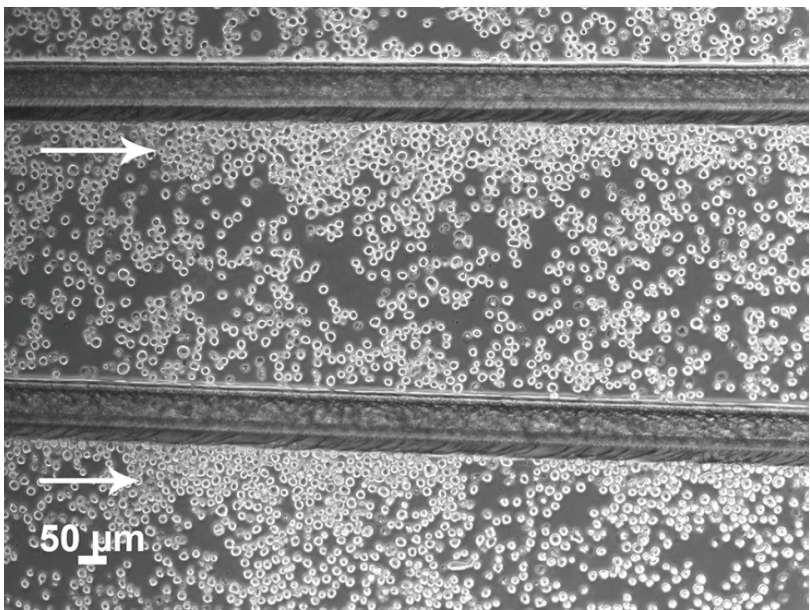

(C)

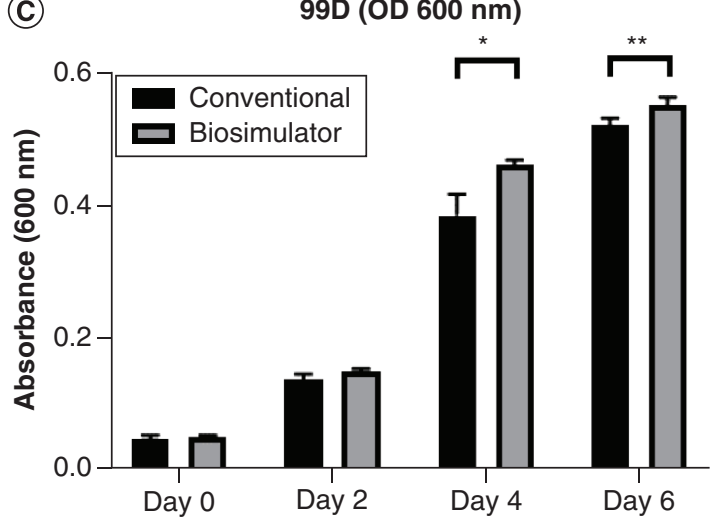

(B)

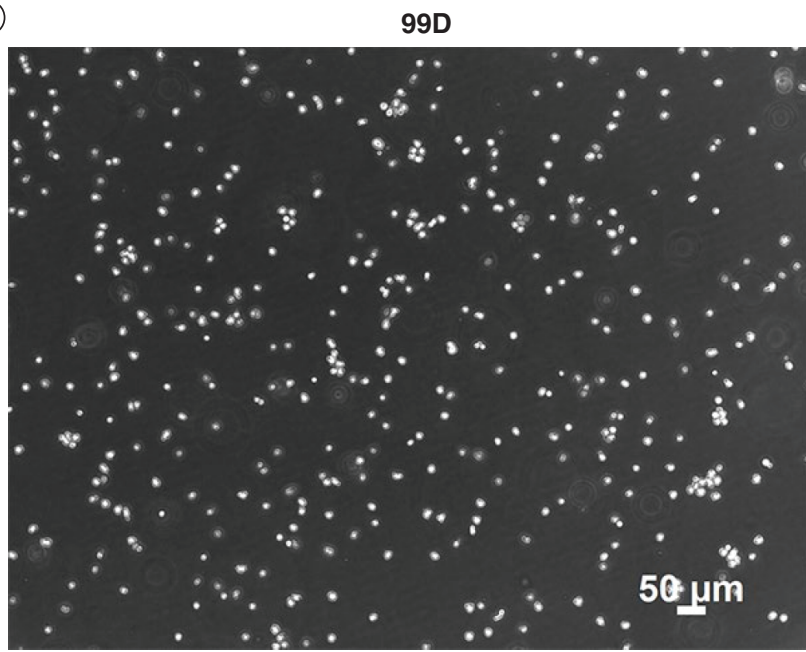

(D)

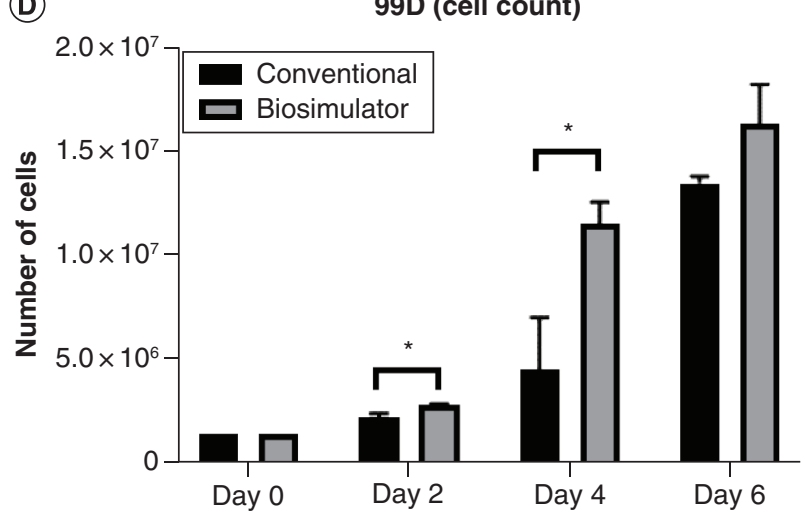

Figure 2. Engraved surface-induced proliferation of 99D nonadherent cells. The hybridoma cell 99D was induced to proliferate by the engraved surface. (A) Photomicrograph showing 99D cells adhering to the engraving of a biosimulator compared with (B) growth in a conventional dish. (C) Absorbance at $600 \mathrm{~nm}$ of 99D cells growing in a biosimulator or conventional dish. (D) Cell count of 99D cells growing in a biosimulator or conventional dish using a hemocytometer. Experiments were repeated five times.

${ }^{*} \mathrm{p}<0.05,{ }^{* *} \mathrm{p}<0.01$ as determined by $t$-test.

need to develop new strategies to induce proliferation of microorganisms. The microbiome is involved in health and diseases [7] and there is a need to determine dysbiotic microorganisms to facilitate diagnosis and treatment.

Samples were cultured from different environments - air, water, soil and host oral cavity - in the biosimulator. The microorganisms were found to adhere to the engravings of the biosimulator within $24 \mathrm{~h}$ (Figure 5). After $48 \mathrm{~h}$, they proliferated and covered the entire biosimulator. Analysis of the soil microbiome by spectrometry demonstrated that the biosimulator induced more proliferation of the microorganisms compared with conventional techniques (Figure 5).

The biosimulator induced proliferation of a large number of Firmicutes in samples from air, soil, water and oral cavity compared with culture using conventional techniques (Figure 6). The phylum Firmicutes includes the class Clostridia and genus Clostridium and consists of a large group of anaerobic to aerotolerant spore-forming bacilli found in soil and in the gut flora of humans and animals. Clostridia include both gram-positive and gram-negative species, although the majority are gram-positive. Interestingly, many grampositive species lose the Gram reaction, resulting in gram-negative cultures [8]. Some species of Clostridium can become aerotolerant on subculture; however, only a few (C. carnis, C. histolyticum, and C. tertium) can grow under aerobic conditions. The vast majority of human oral Clostridia comprises the families Lachnospiraceae, Peptostreptococcaceae and Veillonellaceae [9]. Microbial sequencing demonstrated that the biosimulator induced proliferation of bacterial phyla of air (Figure 7, Supplementary Table 1), water (Figure 8, Supplementary Table 2), soil (Figure 9, Supplementary Table 3) and oral samples (Figure 10, Supplementary Table 4). 


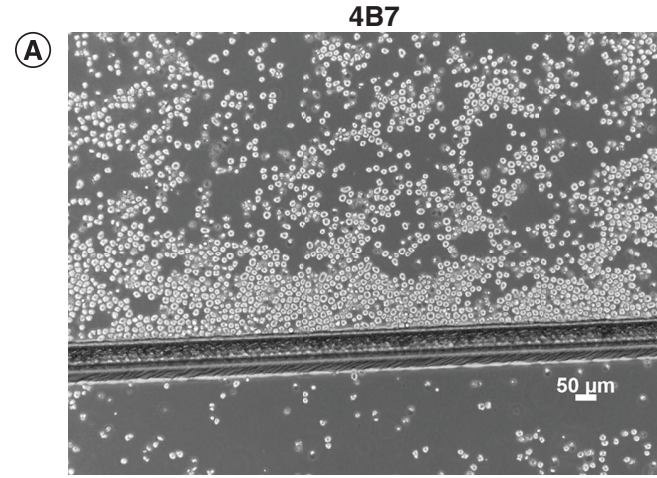

(C)

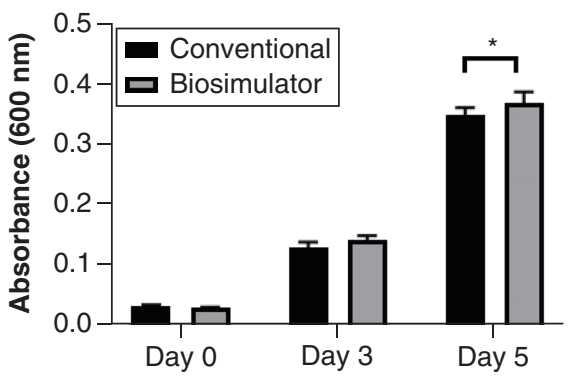

(B)

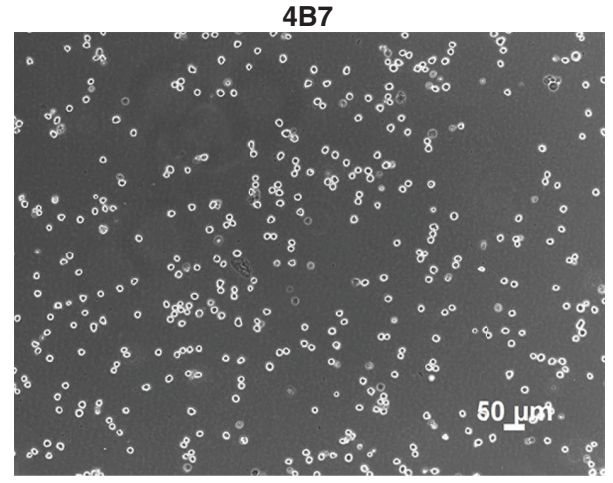

(D) 4B7 (cell count)

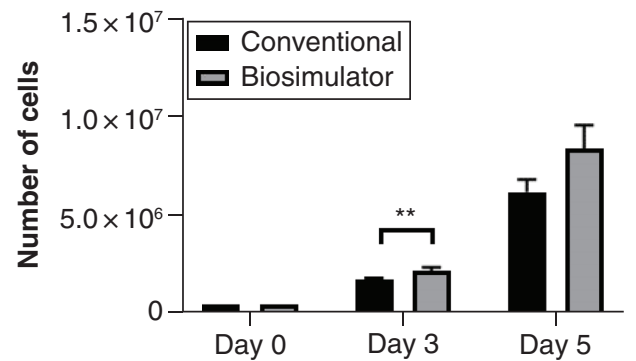

Figure 3. Engraved surface-induced proliferation of the 4B7 nonadherent cells. The hybridoma cell 4B7 was induced to proliferate by the engraved surface. (A) Photomicrograph showing 4B7 cells adhering to the engraving of a biosimulator compared with (B) growth in a conventional dish. (C) Absorbance at $600 \mathrm{~nm}$ of 4B7 cells growing in a biosimulator or conventional dish. (D) Cell count of $4 \mathrm{~B} 7$ cells growing in a biosimulator or conventional dish using a hemocytometer.

${ }^{*} \mathrm{p}<0.05,{ }^{* *} \mathrm{p}<0.01$ as determined by $t$-test.

(A)

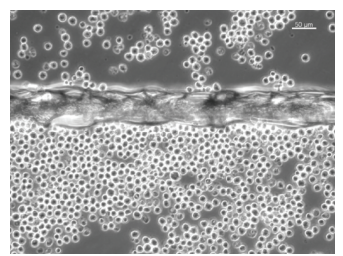

(B)

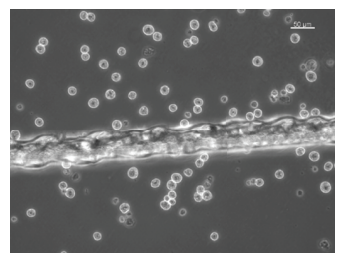

(C)

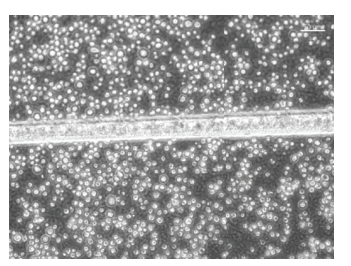

Figure 4. Treatment of nonadherent $4 B 7$ cells with specific inhibitors prevented adhesion of cells to the engraved plastic surface. (A) Nonadherent cells adhering to the engraved plastic surface. (B) Salicylic acid treatment prevented adhesion of the nonadherent cells in an engraved plastic dish. (C) Treatment with pectasol prevented cell adhesion in an engraved plastic dish. Experiments were repeated three times.

Culturing the microbiome of air from the built environment (office) using a biosimulator led to the proliferation of the genera Comamonas, Aeromonas, Bacillus, Clostridium (sensu stricto), Shigella, Escherichia, Klebsiella, Providencia, Serratia, Exiguobacterium, Acinetobacter, Neisseria, Lysinibacillus, Pseudomonas, Shewanella, Lactococcus and Streptococcus. However, these microorganisms were not observed when cultured by conventional techniques.

Culturing the microbiome of spring water using a biosimulator led to the proliferation of the genus Proteus. Culturing the microbiome of soil using a biosimulator led to the proliferation of the genus Comamonas.

The principle of contact guidance or topographic guidance was introduced by Ross Harrison in 1914 [10] and refined by Paul Weiss in 1945, when he demonstrated that cells elongate along the direction of the groove and that migration is influenced by the grooves [11]. The cultured cells exhibit thigmotropism, sensing the surface topography of their environment and reacting to these surface cues. 


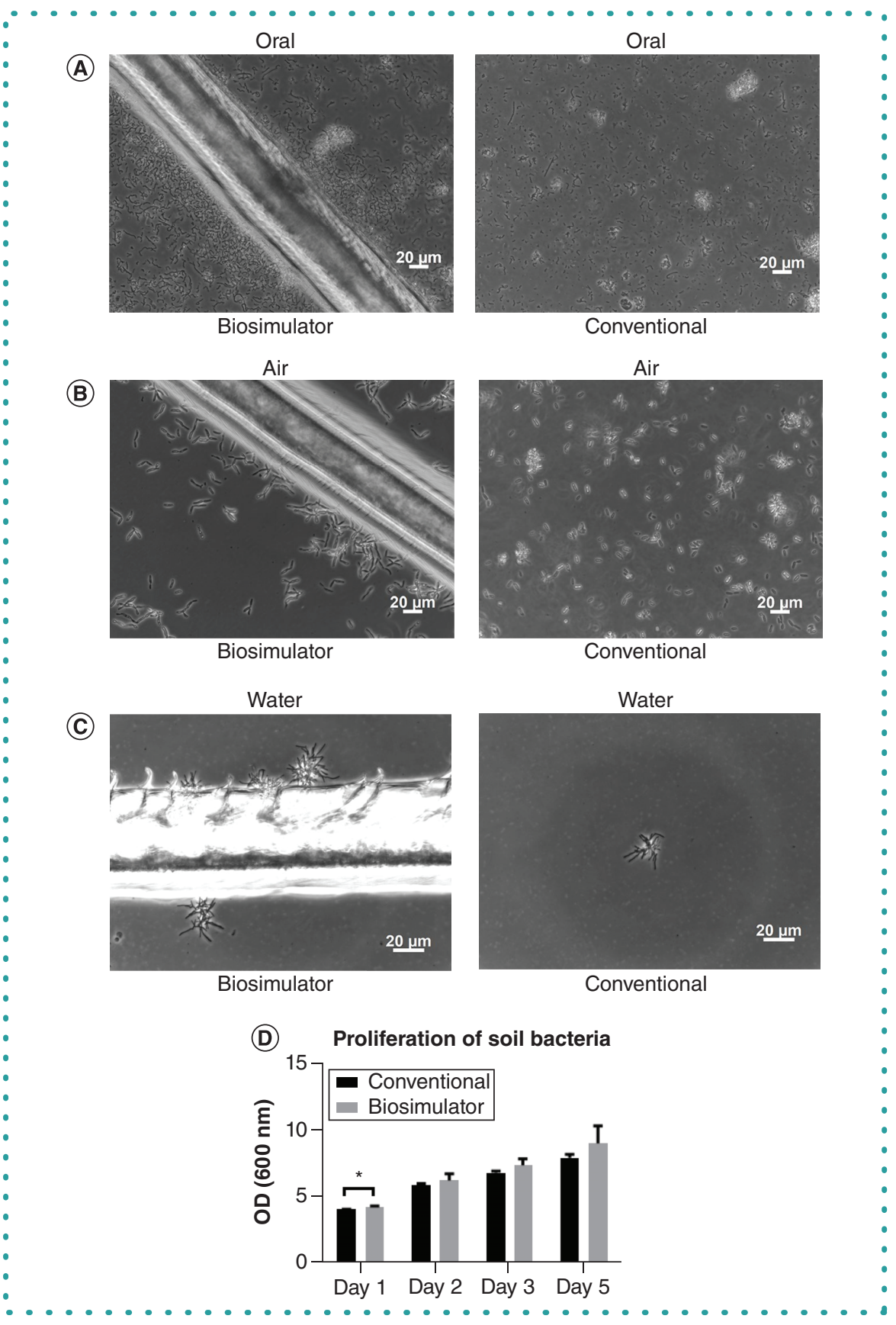

Figure 5. The biosimulator induced proliferation of bacteria from the oral cavity, air, water and soil samples. The engraving in the biosimulator induced proliferation of the bacteria from the (A) oral cavity, (B) air, (C) water and (D) soil samples. Experiments were repeated three times.

Cellular behavior, including cell shape, adhesion, orientation, migration and proliferation, is influenced by surface chemistry and surface topography $[12,13]$.

During cell culture, cells attach to the surface via focal adhesions that connect the surface to the cytoskeleton. The surface chemistry, electrostatic charge, wettability and elastic modulus influence cell growth. The topographical surface provides clues to cell alignment, migration and outgrowth of neurites along a specific orientation. In cell biology studies, to understand the ability of cells to respond to surface topography, grooved substrates are often used. The cells are physically guided along the direction of the grooves, reorganized by the cytoskeleton. However, the mechanism by which cells recognize surface geometry is not clearly understood. The literature suggests it may be a passive process, whereby adhesion molecules best fitting the local topography simply draw cells or cellular components into the observed shape. In several cell lines, surface topography has been shown to be important for the early events of attachment and formation of focal adhesions, activating mechanotransduction events which may eventually determine cell fate and consequent 


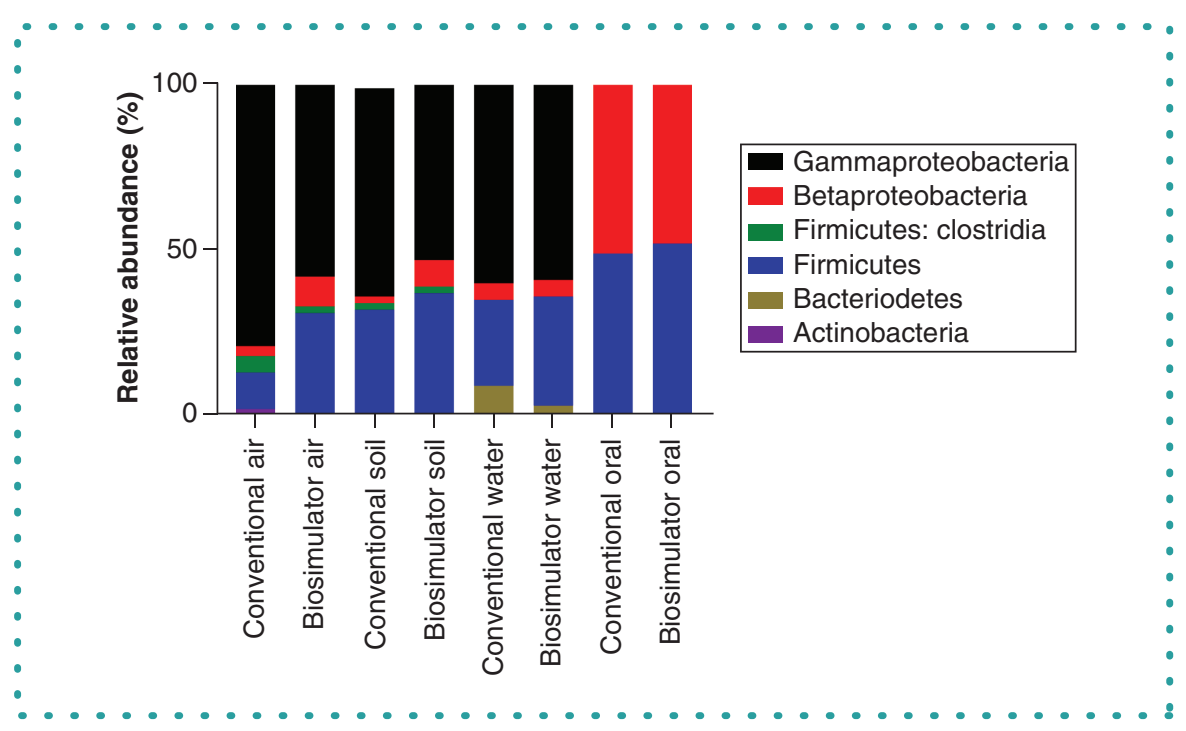

Figure 6. Relative abundance of bacteria at the phylum level cultured in the biosimulator or conventional dishes. Bacteria from air, soil, water and oral cavity samples were cultured in a biosimulator or conventional dish and the taxonomy determined by $16 \mathrm{~S}$ rRNA sequencing. Each bar represents the mean of three samples.

tissue formation. Among different topographies, micrometer-sized grooved surfaces have been extensively studied for their effects on cell alignment because they can be easily fabricated. As an example, culture of osteogenic cells on grooved surfaces results in strong orientation in the direction of the grooves - unlike on flat surfaces, where a random orientation is generally observed. It has also been demonstrated that microgrooves with widths similar to the cell size induce better cell guidance, whereas the guidance is weaker when cells are cultured in grooves with widths larger than the cells [14-16]. Focal adhesions and actin microfilament bundles and microtubules are found to align along micrometer-sized grooves and ridges, whereas, on smooth substrates, no preferred orientation is exhibited by cells and cytoskeletal elements [17]. In the culture of osteoblastic cells on microgrooves with widths ranging from 4 to $38 \mu \mathrm{m}$, it was shown that the narrower grooves (4-16 $\mu \mathrm{m} ; 0.5-2 \times$ cell size) are more effective in guiding the cell orientation. Similarly, it was shown that microgrooves with widths ranging from 2 to $12 \mu \mathrm{m}$ exhibit great contact guidance effects on the shape and orientation of rat bone marrow cells and fibroblasts [16].

Knowledge of the phenomenon that cells have affinity for grooves on the substrate has led to the development of the field of cellular micropatterning. Cellular micropatterning is a tool to accurately design cell-substrate attachment, tissue engineering, biological assays and biosensors, and is used for drug development studies [18]. A broad range of techniques and materials have been employed to fabricate well-defined topographical and chemical cues to assess cell micropatterning. Some of these fabrication techniques are based on photolithography and reactive ion etching that may be followed by anisotropic etching [19]. In recent years, microfluidics encompassing sophisticated micropatterning designs has led to the development of powerful tools for single cell analysis. The localized microenvironment is precisely controlled by microfluidic techniques, thereby increasing accuracy. The technique is important for disease diagnosis and personalized medicine [20].

Micropatterning can be fabricated into microwell arrays to achieve three different dimensions: single cell (1D), cell monolayer (2D) and cell spheroid (3D). As cells in the in vivo environment are surrounded by other cells in three dimensions, 2D cell culture does not adequately take into account the natural environment of cells. As a result, 2D cell culture tests sometimes provide misleading and nonpredictive data for in vivo responses [21]. 3D in vitro cell models provide a more realistic cellular environment and permit the reproduction of in vivo cellular phenotypes [22]. Spheroids realistically reflect in vivo cell behaviors and provide results more closely aligned to those of in vivo tests, thereby making them useful in drug developmental studies [21].

$3 D$ cell culture is used in stem cell and tumor cell research [23,24]. In 3D cell culture, human embryonic stem cells (hESCs) have the potential to differentiate into over 200 diversely functioning cell types. Microwell culture permits generation of hESC colonies with a defined size that can later form monodisperse embryoid bodies. When cultured in this system, hESCs retain pluripotency and self-renewal, and can be passaged to standard unconstrained culture conditions [23]. At the intersection between tissue engineering and oncology, $3 \mathrm{D}$ in vitro tumor models simulate the in vivo physiological microenvironment. Working in 3D involves the formation of spheroids - aggregates that can either be grown in suspension, encapsulated, or grown on top of a 3D matrix using different 3D methods [25]. Many cell lines show a reduced proliferation rate in 3D cultures compared with those cultured in 2D. Furthermore, many currently available 3D cell culture techniques are slow, time-consuming, expensive and lack reproducibility [21].

In the initial days of culture in the biosimulator, the pattern of the engraving influenced the nature of cell adherence: the cells were more adhered on the outer edge of the engraved line than the inner edge. It is not understood why the cells show polarity when cultured 


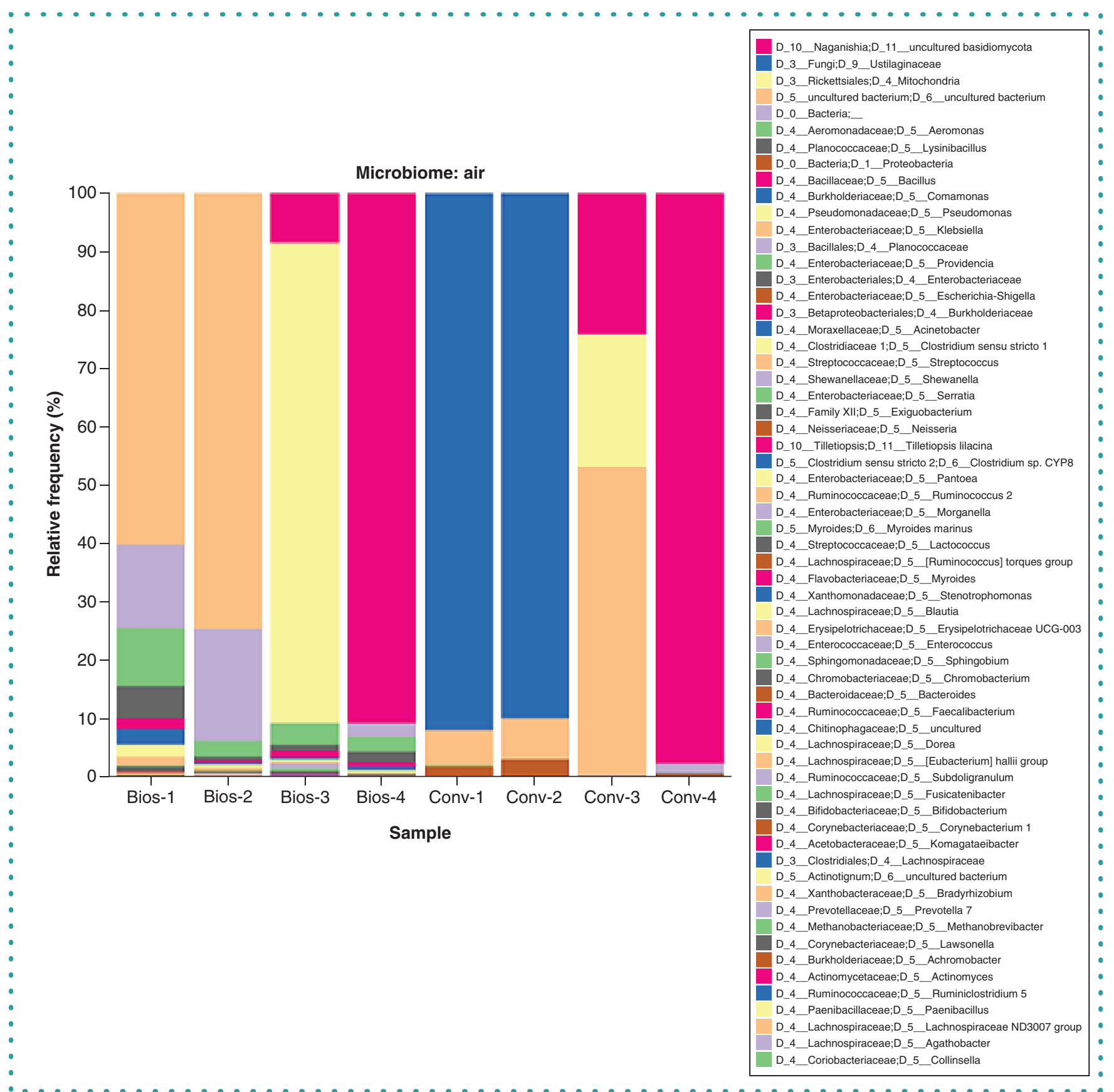

Figure 7. Relative abundance of microorganisms of air (bacteria and fungi) at the genus level cultured in the biosimulator or conventional dishes. Bacteria from air (office) were cultured in a biosimulator or conventional dish for $48 \mathrm{~h}$ and the taxonomy determined by $16 \mathrm{~S}$ rRNA sequencing ( $\mathrm{n}=4 \mathrm{per}$ treatment).

on a dish with parallel engravings; however, the physical features influencing the cells to form patterns include the circular shape of the Petri dish and the engravings on the hydrophilic plastic surface.

The engraving on the plastic surface did not induce very strong affinity to the cells, which had only a weak adhesion to the engravings. Rapid shaking and extreme washing prevented adhesion of the nonadherent cells. Nevertheless, the adhesion induced proliferation of eukaryotic and prokaryotic cells.

The fundamental units of cell adhesion are protein complexes consisting of three classes of proteins: cell adhesion molecules/adhesion receptors, extracellular matrix proteins and cytoplasmic plaque/peripheral membrane proteins. The cell adhesion proteins include members of the integrin, cadherin, immunoglobulin, selectin and proteoglycan super families [26]. We have not deter- 


\section{Microbiome: water}

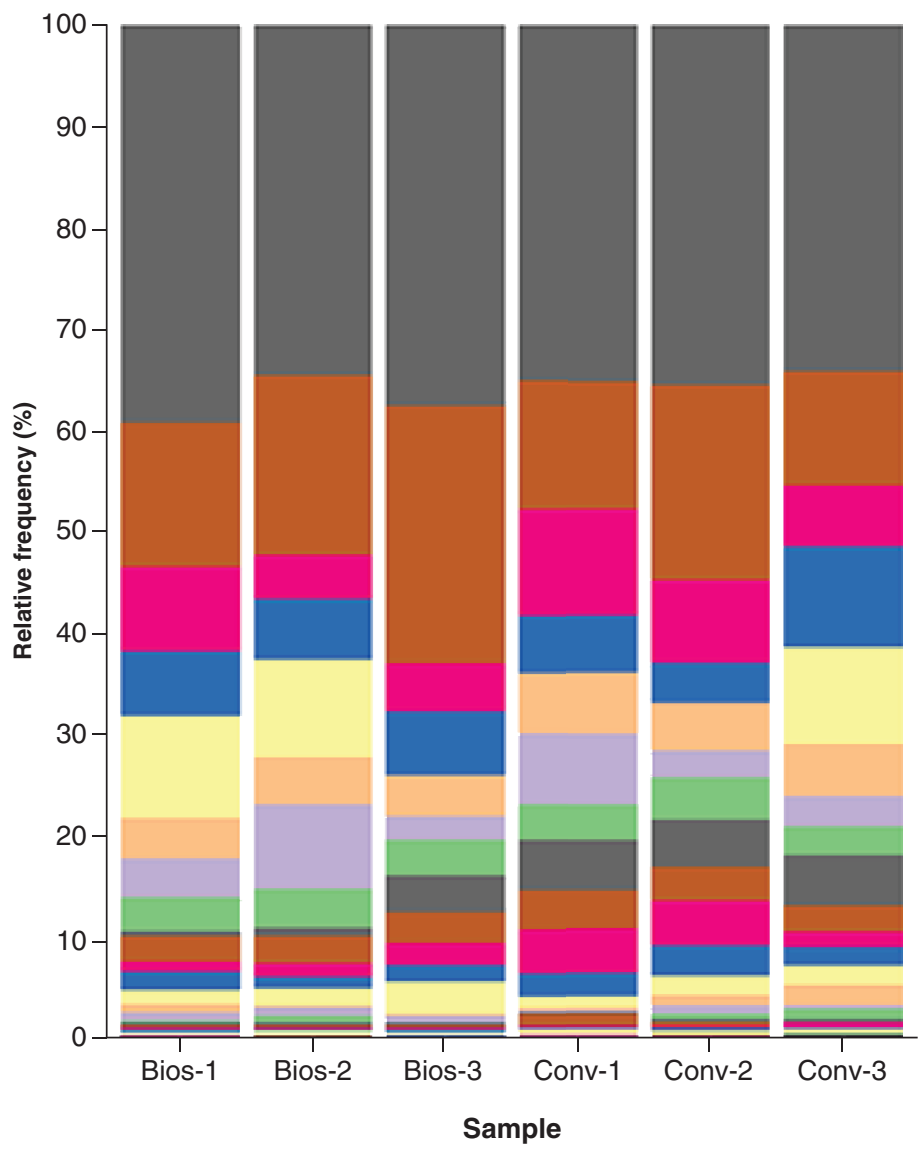

D_4_Aeromonadaceae;D_5_Aeromonas

D_4_Bacillaceae;D_5_Bacillus

D_4_Planococcaceae;D_5_Lysinibacillus

D_4_Enterobacteriaceae;D_5_Providencia

D_3_Bacillales;D_4_Planococcaceae

D_4_Enterobacteriaceae;D_5_Klebsiella

D_4_Burkholderiaceae;D_5_Comamonas

D_3_Enterobacteriales;D_4_Enterobacteriaceae

D_5_Myroides;D_6_Myroides marinus

D 4 _Moraxellaceae;D 5 Acinetobacter

D_4_Flavobacteriaceae;D_5_Myroides

D_3_Betaproteobacteriales;D_4_Burkholderiaceae D_4_Pseudomonadaceae;D_5_Pseudomonas

D_4_Shewanellaceae;D_5_Shewanella

D_4_Family XII;D_5_Exiguobacterium

D_4_Enterococcaceae;D_5_Vagococcus

D_4_Enterobacteriaceae;D_5_Pantoea

D_5_Myroides;D_6_Myroides sp. OTU s6b

D_4_Enterobacteriacee;D_5_Morganella

D_4_Enterobacteriacee;D_5_Serratia

D_4_Streptococcaceae;D_5_Lactococcus

D_4_Enterobacteriaceae;D_5_Escherichia-Shigella

D_4_Xanthomonadaceae;D_5_Stenotrophomonas

D_4_Enterococacceae;D_5_Enterococcus

D_2_Bacilli;D_3_Bacillales

D_4_enterobacteriaceae;D_5_Proteus

D_4_Chromobacteriaceae;D_5_Chromobacterium

D_4_Weeksellaceae;D_5_Chryseobacterium

D_4_Vibrionaceae;D_5_Vibrio

D_4_Hydrogenophilacease;D_5_Thiobacillus

D_4_Enterobacteriaceae;D_5_Hafnia-Obesumbacterium

D_4_Weeksellaceae;D_5_Empedobacter

D_4_Corynebacteriaceae;D_5_Lawsonella

D_5_Joenoides;D_6_Joenoides intermedia

D_5_Paenibacillus;D_6_Paenibacillus taiwanensis

D_4_Clostridiaceae 1;D_5_Clostridium sensu stricto 1

D_4_Neisseriaceae;D_5_Neisseria

D_4_Sphingobacteriaceae;D_5_Sphingobacterium

D_5_Paenibacillus;D_6_Paenibacillus sp. St-s

D_5_Empedobacter;D_6_Empedobacter sp. PH7-1

D_5_uncultured;D_6_bacterium DCE29

D_3_Lactobaciliales;D_4_Enterococcaceae

DD_4_Nitrosomonadaceae;D_5_MND1

D_4_Burkholderiaceae;D_5_Alcaligenes

D_5_Microcoleus PCC-7113;D_6__Microcoleus sp. HTT-U-KK5

D_4_Propionibacteriaceae;D_5_Cutibacterium

D_4_Chromobacteriaceae;D_5_Vogesella

D_0_Bacteria; _

Figure 8. Relative abundance of microorganisms of water at the genus level cultured in the biosimulator or conventional dishes. Bacteria from spring water were cultured in a biosimulator or conventional dish for $48 \mathrm{~h}$ and the taxonomy determined by $16 \mathrm{~S}$ rRNA sequencing $(\mathrm{n}=3$ per treatment). 


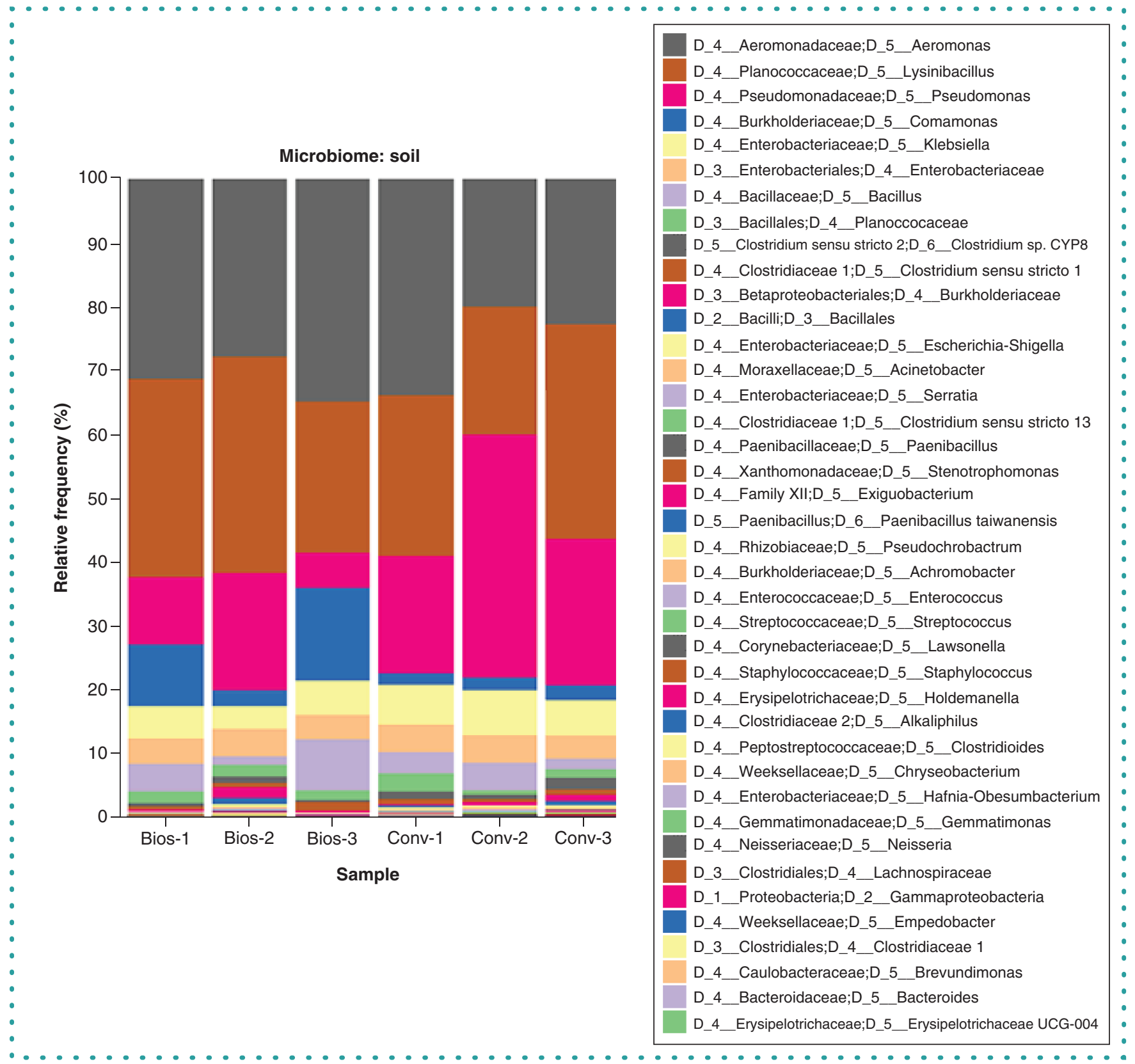

Figure 9. Relative abundance of microorganisms of soil at the genus level cultured in the biosimulator or conventional dishes. Bacteria from soil were cultured in a biosimulator or conventional dish for $48 \mathrm{~h}$ and the taxonomy determined by 16S rRNA sequencing $(\mathrm{n}=3$ per treatment).

mined which adhesion molecules were expressed on the nonadherent cells when cultured in a biosimulator. However, we determined whether inhibiting the expression of the adherent proteins would prevent the nonadherent cells from adhering to the engraving.

For these inhibition studies, we used salicylic acid and pectasol. It is known that salicylic acid prevents adhesion of eukaryotic and microbial cells [27-31]. The adhesion is mediated by ERK signaling and the inhibition of this signaling prevents adhesion [32,33]. For example, coating urinary catheters with salicylic acid reduces bacterial adherence and the risk of urinary tract infection [30].

Pectasol-C is a modified pectin compound used in the inhibition of adhesion and metastasis in cancer cells [6]. Galectin-3 (Gal-3) is a $29-\mathrm{kDa} \beta$-galactoside-binding adhesion protein and is expressed intracellularly and extracellularly by various cell types. Pectasol is known to inhibit Gal-3, thereby inhibiting adhesion [34]. In this study, we used both salicylic acid and pectasol for inhibition studies. The cells did not adhere to the engravings of the biosimulator in the presence of salicylic acid and pectasol, demonstrating that the adhesion was mediated by adhesion proteins.

Biofouling is a limiting factor in medical devices. When associated with the biological environment, biomedical devices are prone to surface biofouling due to adhesion of microbial or thrombotic agents [35]. We demonstrated that engravings could predict where 


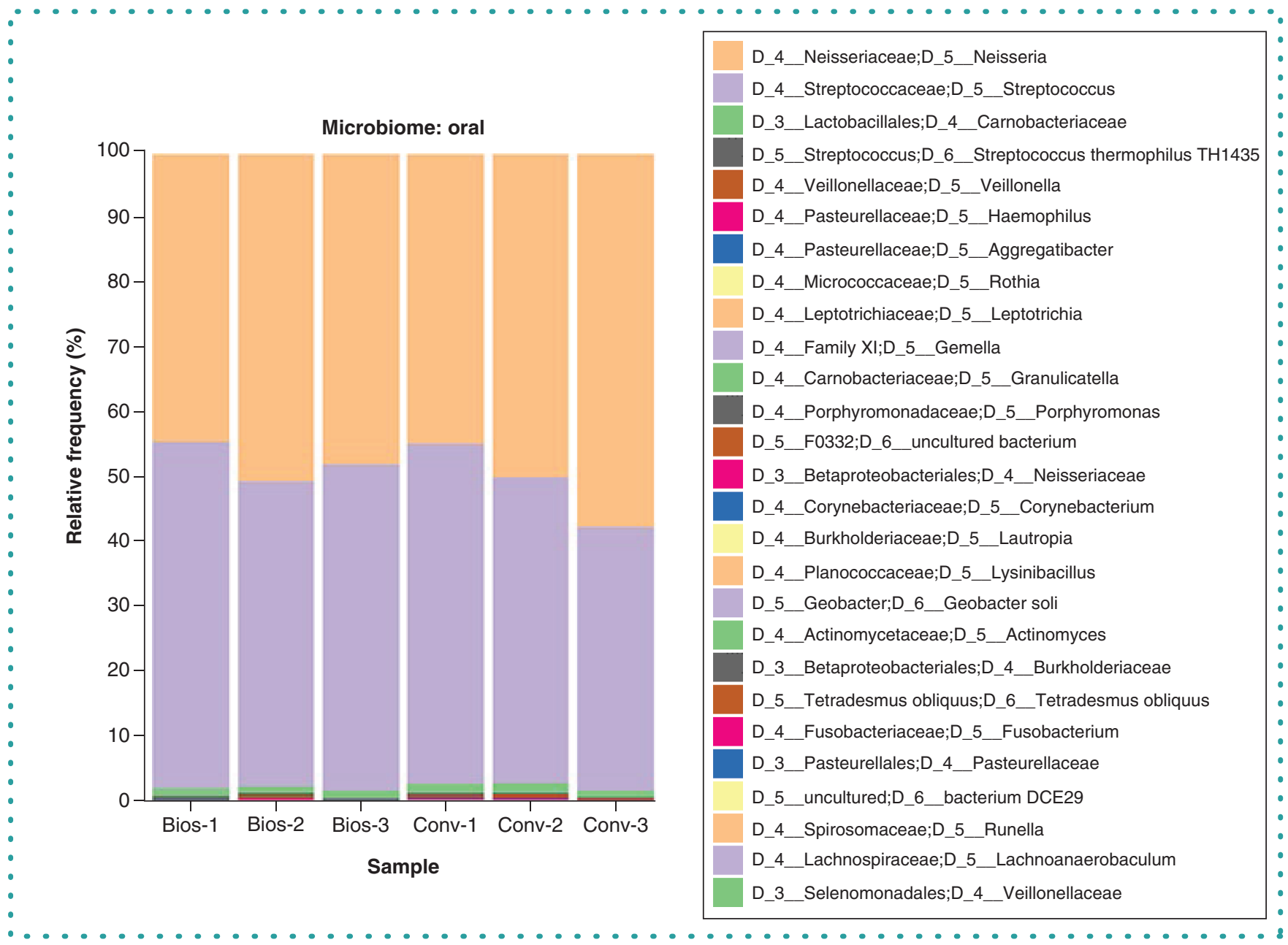

Figure 10. Relative abundance of microorganisms of the oral cavity at the genus level cultured in the biosimulator or conventional dishes. Bacteria from the oral cavity were cultured in a biosimulator or conventional dish for $48 \mathrm{~h}$ and the taxonomy determined by $16 \mathrm{~S}$ rRNA sequencing $(\mathrm{n}=3$ per treatment).

nonadherent cells adhere. Based on this property, we could design better probes for biomedical applications that could resist biofouling. The phenomenon thus has wide applications in biomedical engineering.

Use of animal models is the preferred method for drug development studies, and single cell analysis provides key information critical to understanding disease processes. However, animal models are expensive and cumbersome, and the researcher has to analyze and collect data of single cells from a pool of cells. Current strategies do not allow analysis of single cells without removing them from the context of interest, which not only destroys contextual information but also may impair the process under study [36]. The induction of adherence in nonadherent cells has wide application in cell biology. The phenomenon could be used to study the mechanism by which autoreactive T cells adhere to pancreatic $\beta$ cells. Similarly, the mechanism by which monocytes or macrophages adhere to artery walls could be studied using a biosimulator. We have demonstrated that pattern formation can be prevented using drugs that prevent cell adhesion. Based on this principle, new drugs could be tested for their ability to prevent adhesion in different cells. Promising drug candidates that inhibit adhesion molecules in autoreactive T cells could help to combat diseases such as Type I diabetes. Use of the biosimulator to aid development of novel drugs by inhibiting adhesion of nonadherent cells is a more cost-effective strategy than using animal models.

The majority of microorganisms found in nature, including pathogens infecting humans, animals and plants, are nonculturable using conventional techniques [37]. A device that could be used to induce proliferation of these microorganisms will be beneficial in diagnostics as well as the development of new drugs and vaccines.

There have been few modifications in the design and construction of the Petri dish since its development in the 19th century by the German bacteriologist Julius Petri. Surface modified (hydrophilic) polystyrene plates are in vogue for cell culture studies. When adherent cells are cultured on a Petri dish, they spread rapidly and grow to confluence within a couple of days (the exact time required to form confluence depends on the nature of the cell line). However, when nonadherent cells are cultured, some cells attach to the bottom of 
the Petri dish, but the majority remain suspended in the medium. Currently, there are no specialized plates for transforming nonadherent cells to adherent cells.

In this paper, it is demonstrated that the biosimulator is a 2D cell system. Most of the 2D systems in the literature are designed for adherent cells; we developed the biosimulator for nonadherent cells and the microbiome. The biosimulator does not have any special coating for inducing adherence. The only variable is the engraving that induces adhesion and proliferation of eukaryotic cells and the microbiome. The width of the engraving is larger than the nonadherent cells. Unlike the adherent cells that formed lamellopodia in the grooves, there was no lamellopodia formation in the nonadherent cells attached to the engravings, resulting in weak affinity of the cells to the engravings. The purpose of the biosimulator is to induce adhesion of nonadherent cells so that they could be used for drug developmental studies; however, further work is required to determine whether induction of adhesion by the biosimulator changes the properties of the nonadherent cells. In addition to induction of adhesion in nonadherent cells, the biosimulator also induced adhesion of microorganisms, and thus could also be used to monitor the microbiome of host and environmental samples.

We used the biosimulator to induce proliferation of microorganisms from different environments: air, water, soil and host. The microorganisms from the environmental samples proliferated within $24 \mathrm{~h}$ in a biosimulator. In the first $24 \mathrm{~h}$, we observed that the bacteria had more affinity to the engraving; after $24 \mathrm{~h}$ the whole biosimulator was covered with bacteria. To determine if there were changes in the microbial species after culture in different conditions, we cultured the environmental samples in a biosimulator as well as a conventional Petri dish. Microbial sequencing demonstrated that the biosimulator could induce proliferation of microorganisms better than culturing in conventional dishes; we observed several species of bacteria in the environmental samples cultured in a biosimulator that were not seen in those cultured in conventional dishes. We hypothesize that the biosimulator may be useful in encouraging the proliferation of difficult-to-culture microorganisms.

To conclude, the induction of adhesion and proliferation of nonadherent cells and microorganisms on an engraved plastic surface will open new avenues of research in immunology, cell biology and microbiology.

\section{Supplementary data}

To view the supplementary data that accompany this paper please visit the journal website at: www.futurescience.com/doi/suppl/10.2144/btn-2020-0022

\section{Author contributions}

S Thomas conceived, designed and performed the experiments, analyzed the data, and wrote the paper.

\section{Acknowledgments}

The author thanks MA Azcarate-Peril and J Roach of the University of North Carolina Microbiome Core for microbiome analysis.

\section{Financial \& competing interests disclosure}

The author wishes to acknowledge receiving grants and support from the Wawa Foundation, Abraham Thomas Foundation and Women's Board of Lankenau. The UNC Microbiome Core is funded partially by NIH/NIDDK grant number P30 DK34987. The author has no other relevant affiliations or financial involvement with any organization or entity with a financial interest in or financial conflict with the subject matter or materials discussed in the manuscript apart from those disclosed.

No writing assistance was utilized in the production of this manuscript.

\section{Open access}

This work is licensed under the Attribution-NonCommercial-NoDerivatives 4.0 Unported License. To view a copy of this license, visit http: //creativecommons.org/licenses/by-nc-nd/4.0/

\section{References}

1. Wu CY, Stoecklein D, Kommajosula A et al. Shaped 3D microcarriers for adherent cell culture and analysis. Microsyst. Nanoeng. 4, 21 (2018).

2. Barcina I, Arana I. The viable but nonculturable phenotype: a crossroads in the life-cycle of non-differentiating bacteria? Rev. Environ. Sci. Biotechnol. 8, 245-255 (2009).

3. Li L, Mendis N, Trigui H, Oliver JD, Faucher SP. The importance of the viable but nonculturable state in human bacterial pathogens. Front. Microbiol. 5, 258 (2014).

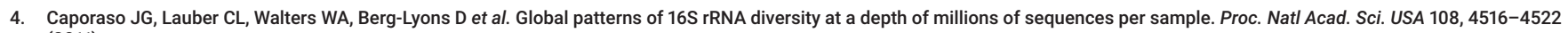
(2011).

5. Cao Y, DuBois DC, Almon RR, Jusko WJ. Pharmacokinetics of salsalate and salicylic acid in normal and diabetic rats. Biopharm. Drug Dispos. 33, 285-291 (2012).

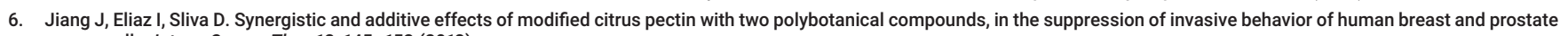
cancer cells. Integr. Cancer Ther. 12, 145-152 (2013).

7. Thomas S, Izard J, Walsh E et al. The host microbiome regulates and maintains human health: a primer and perspective for non-microbiologists. Cancer Res. 77, 1783-1812 (2017).

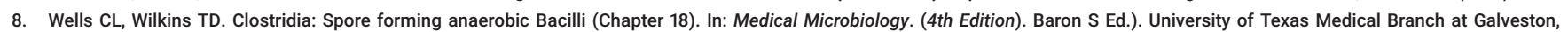
Galveston, TX (1996)

9. Dewhirst FE, Chen T, Izard J, Paster BJ et al. The human oral microbiome. J. Bacteriol. 192, 5002-5017 (2010).

10. Harrison RG. The reaction of embryonic cells to solid structures. J. Exp. Zool. 17, 521-544 (1914).

11. Weiss P. Experiments on cell and axon orientation in vitro: the role of colloidal exudates in tissue organization. J. Exp. Zool. 100, 353-386 (1945). 
12. Hamilton DW, Oakley C, Jaeger NA, Brunette DM. Directional change produced by perpendicularly-oriented microgrooves is microtubule-dependent for fibroblasts and epithelium. Cell Motil. Cytoskeleton 66, 260-271 (2009).

13. Li Q, Guo Y, Wang Y. The influence of microgrooved surfaces on the behavior and cellular function of osteoblasts. Dent. Oral Craniofac. Res. 2. doi: 10.15761/DOCR.1000176 2016) (Epub ahead of print).

14. Zhou F, Yuan L, Huang H, Chen H. Phenomenon of "contact guidance" on the surface with nano-micro-groove-like pattern and cell physiological effects. Chin. Sci. Bull. 54 , $3200-3205$ (2009).

15. Peterbauer T, Yakunin S, Siegel J et al. Dynamics of spreading and alignment of cells cultured in vitro on a grooved polymer surface. J. Nanomater. 2011 Article ID: 413079 (2011).

16. Sun L, Pereira D, Wang Q et al. Controlling growth and osteogenic differentiation of osteoblasts on microgrooved polystyrene surfaces. PLoS ONE 11(8), e0161466 (2016).

17. Teixeira Al, Abrams GA, Bertics PJ, Murphy CJ, Nealey PF. Epithelial contact guidance on well-defined micro- and nanostructured substrates. J. Cell Sci. 116(Pt 10), 1881-1892 (2003).

18. Tourovskaia A, Barber T, Wickes BT et al. Micropatterns of chemisorbed cell adhesion-repellent films using oxygen plasma etching and elastomeric masks. Langmuir 19 , $4754-4764$ (2003).

19. Fernández-Castillejo S, Formentín P, Catalán Ú, Pallarès J, Marsal LF, Solà R. Silicon microgrooves for contact guidance of human aortic endothelial cells. Beilstein J. Nanotechnol. 8, 675-681 (2017).

20. Gao D, Jin F, Zhou M, Jiang Y. Recent advances in single cell manipulation and biochemical analysis on microfluidics. Analyst 144, 766-781 (2019).

21. Edmondson R, Broglie JJ, Adcock AF, Yang L. Three-dimensional cell culture systems and their applications in drug discovery and cell-based biosensors. Assay Drug Dev. Technol. 12, 207-218 (2014).

22. Park J, Müller M, Kim J, Seidel H. Fabrication of a cell-adhesive microwell array for 3-dimensional in vitro cell model. Biomed. Eng. Lett. 5, 140-146 (2015).

23. Mohr JC, de Pablo JJ, Palecek SP. 3-D microwell culture of human embryonic stem cells. Biomaterials 27, 6032-6042 (2006).

24. Lv D, Hu Z, Lu L, Lu H, Xu X. Three-dimensional cell culture: a powerful tool in tumor research and drug discovery. Oncol. Lett. 14, 6999-7010 (2017).

25. Hoarau-Véchot J, Rafii A, Touboul C, Pasquier J. Halfway between 2D and animal models: are 3D cultures the ideal tool to study cancer-microenvironment interactions? Int. J. Mol. Sci. 19(1), pii: E181 (2018).

26. Gumbiner BM. Cell adhesion: the molecular basis of tissue architecture and morphogenesis. Cell 84, 345-357 (1996).

27. Gerli R, Gresele P, Bistoni $O$ et al. Salicylates inhibit T cell adhesion on endothelium under nonstatic conditions: induction of L-selectin shedding by a tyrosine kinase-dependent mechanism. J. Immunol. 166, 832-840 (2001).

28. Eisele G, Schwedhelm E, Schieffer B, Tsikas D, Boger RH. Acetylsalicylic acid inhibits monocyte adhesion to endothelial cells by an antioxidative mechanism. J. Cardiovasc. Pharmacol. 43, 514-521 (2004).

29. Weber C, Erl W, Pietsch A, Weber PC. Aspirin inhibits nuclear factor-kappa B mobilization and monocyte adhesion in stimulated human endothelial cells. Circulation 91 , 1914-1917 (1995).

30. Farber BF, Wolff AG. The use of salicylic acid to prevent the adherence of Escherichia coli to silastic catheters. J. Urol. 149, 667-670 (1993).

31. Bandara BM, Sankaridurg PR, Willcox MD. Non-steroidal anti inflammatory agents decrease bacterial colonisation of contact lenses and prevent adhesion to human corneal epithelial cells. Curr. Eye Res. 29, 245-251 (2004).

32. Pillinger MH, Capodici C, Rosenthal P et al. Modes of action of aspirin-like drugs: salicylates inhibit erk activation and integrin-dependent neutrophil adhesion. Proc. Nat/ Acad. Sci. USA 95, 14540-14545 (1998).

33. Tanimura S, Takeda K. ERK signalling as a regulator of cell motility. J. Biochem. 162, 145-154 (2017).

34. Hossein G, Keshavarz M, Ahmadi S, Naderi N. Synergistic effects of PectaSol-C modified citrus pectin an inhibitor of Galectin-3 and paclitaxel on apoptosis of human SKOV-3 ovarian cancer cells. Asian Pac. J. Cancer Prev. 14, 7561-7568 (2013).

35. Harding JL, Reynolds MM. Combating medical device fouling. Trends Biotech. 32, 140-146 (2014).

36. Sarkar A, Kolitz S, Lauffenburger DA, Han J. Microfluidic probe for single-cell analysis in adherent tissue culture. Nature Commun. 5, 3421 (2014).

37. Stewart EJ. Growing unculturable bacteria. J. Bacteriol. 194, 4151-4160 (2012). 
